\title{
A Transformer with Increased Leakage Flux for Didactic Considerations on Magnetic Circuits
}

Preliminary Communication

\author{
Hrvoje Vida \\ J. J. Strossmayer University of Osijek \\ Faculty of Electrical Engineering, Computer Science \\ and Information Technology Osijek \\ Kneza Trpimira 2B, Osijek, Croatia \\ hrvoje.vida@etfos.hr
}

\section{Željko Hederić}

J. J. Strossmayer University of Osijek

Faculty of Electrical Engineering, Computer Science and Information Technology Osijek

Kneza Trpimira 2B, Osijek, Croatia

zeljko.hederic@ferit.hr

\author{
Željko Špoljarić \\ J. J. Strossmayer University of Osijek \\ Faculty of Electrical Engineering, Computer Science \\ and Information Technology Osijek \\ Kneza Trpimira 2B, Osijek, Croatia \\ zeljko.spoljaric@ferit.hr

\section{Tin Benšić}
J. J. Strossmayer University of Osijek
Faculty of Electrical Engineering, Computer Science and Information Technology Osijek
Kneza Trpimira 2B, Osijek, Croatia
tin.bensic@ferit.hr

\begin{abstract}
In this paper, we present basics of modeling of complex magnetic circuits in electromagnetic devices for the purpose of didactic considerations. In order to help students better understand the physical issues in a magnetic field, an elementary example of the magnetic field in the coil depending on magnetic core construction has been given. The use of modern software tools for numerical calculation of magnetic fields (FEM) provides easier visualization of the problem and calculation of magnetic circuit parameters for magnetic circuit lumped models by using reluctance. The paper presents an educational scale model of the three-phase three-winding transformers with specially designed coils to provide increased leakage magnetic fluxes and spaces for magnetic field measuring probes.
\end{abstract}

Keywords - finite elements modeling, leakage flux, lumped model, magnetic circuits

\section{INTRODUCTION}

Electromagnetic devices are omnipresent in almost all areas of life. It is very important to understand physical phenomena occurring in technically very complex devices.

For the purpose of didactic considerations in the field of electromagnetism, a transformer with special winding construction was designed. The idea was to enable students through measurements of magnetic fields in different places within the structure of a transformer to calculate the parameters for a model of part of the transformer magnetic circuit. Transformer modeling has been researched for a longer period of time. In this period, different methods for simplifying magnetic circuits have been used, as in [1-6].

Theoretical terms and values describing the magnetic field are as visual as those in electric circuits. Thus, with some simplification, models of magnetic circuits with concentrated parameters (the lumped model) are made by analogy with electric circuits.

Generation of a uniform magnetic field for various practical applications is given and explained in [7-10].
Generation of a uniform magnetic field by using the Helmholtz coils system with an analytical approach and by using ANSYS simulation for better visual presentation of the problem for numerical calculation of magnetic fields (FEM) is given in [11].

Magnetic circuit modeling for the purpose of optimal sizing of an electrical machine in a hybrid electrical vehicle is given in [12]. For the purpose of modeling the electromagnetic linear actuator [13] an equivalent magnetic circuit is also used. The leakage flux in a multi-winding transformer is modeled in [14] for different types of transformers and different air gaps between the core and the winding where an equivalent magnetic circuit model is also used. In [15], a function of relative permeability on frequency and flux density is presented for the purpose of analytical calculations of core losses in electrical machines.

The basis of the magnetic circuit comprises sources (current coil), conductors (magnetic core) and loads (air gaps). By analogy with electric circuits, the magnetic flux through the magnetic core is viewed (presented) as a magnetic current. The source that establishes a magnetic flux is called magnetomotive force (MMF). It is proportional to the coil excitation current $I(\mathrm{~A})$ and 
the number of turns $N$ (ampere-turns). The MMF opposes and holds the balance to magnetic voltage drops on the loads $\mathrm{H} \cdot l(\mathrm{~A})$.

The magnetic core is not an ideal element (analogous to a wire in an electric circuit) through which the magnetic flux passes and it generates losses measured by a magnetic voltage drop. For the purpose of complete analogy, the concept of magnetic resistance (reluctance) has been introduced and a definition of Ohm's law for the magnetic field is given as [16]:

$$
\Theta=\Phi R_{\mathrm{m}}=U_{\mathrm{m}}
$$

where the analogy between magnetic resistance and joining resistance connected in series or parallel is valid.

As in the case of standard electric circuits, the definition of resistance is defined for a flux tube (space with a homogeneous field, a flux, the given cross section and length, a voltage drop and material properties), as shown in Figure 1.

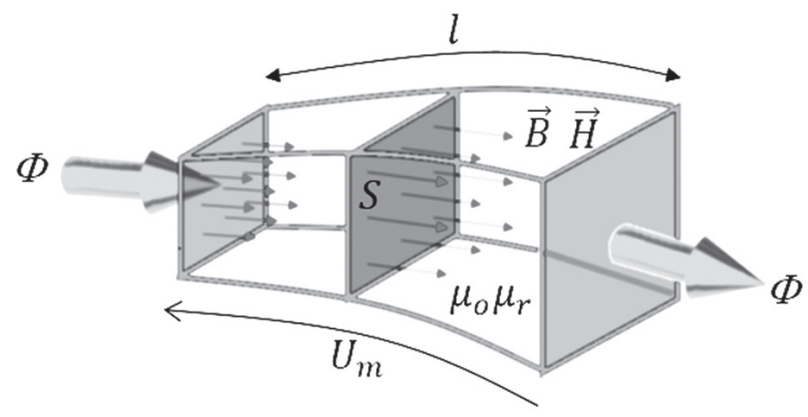

Fig. 1. Magnetic field flux tube - space with a homogeneous field for the definition of reluctance

Resistance is defined as the ratio between the voltage drop and current. In a magnetic field, magnetic resistance is defined as the ratio of the magnetic voltage and the magnetic flux. Because of the constant value of field strength and magnetic inductance, reluctance can be defined by using construction measures and material property values as given in equation [16]:

$$
R_{\mathrm{m}}=\frac{U_{\mathrm{m}}}{\Phi}=\frac{\int \vec{H} \mathrm{~d} \vec{l}}{\iint \vec{B} \mathrm{~d} \vec{S}}=\frac{H \cdot l}{B \cdot S}=\frac{l}{\mu \cdot S}
$$

\section{MAGNETIC CIRCUIT MODELING}

A simple magnetic circuit is composed of a magnetic core, which is closed in the loop without air gaps, and the excitation coil. For simplicity, we will only consider the case of excitation with the current (I) of fixed value.

Figure 2 shows an analogy with electric circuits and modeling of a simple magnetic circuit by using the socalled lumped model with concentrated parameters.

Equations describing a simple magnetic circuit with lumped model [16] are given as:

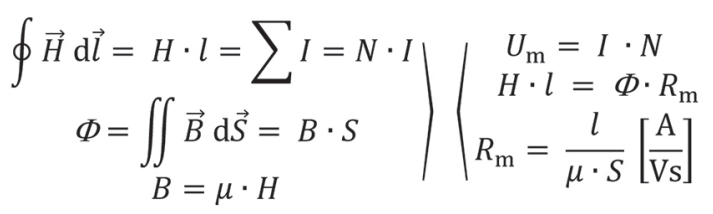

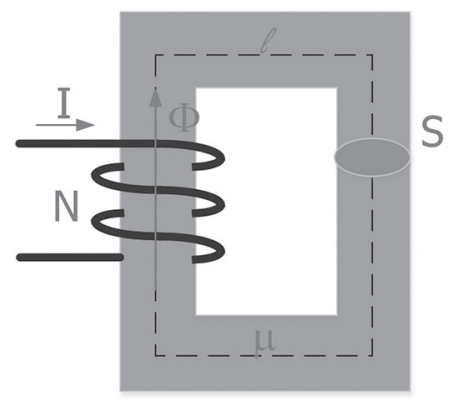

$\Phi$

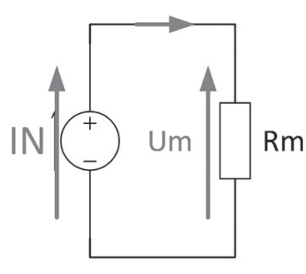

Fig. 2. A simple magnetic circuit and the lumped model with reluctance

However, the model presented previously and derived formulas are valid with one very important assumption that the total magnetic flux, which is caused by the current passing through the turns of the excitation coil, passes only through the magnetic core.

This claim is sufficiently accurate assuming that relative permeability is very large $\left(\mu_{\mathrm{r}}>>1\right)$, which is in reality very rarely fulfilled. Most good magnetic materials have a very non-linear characteristic.

In reality, turns of the coil create magnetic fluxes which are summed and form the total magnetic flow. Such distribution of the magnetic flux has a part which embraces all turns (a linked magnetic flux: $\Psi=N \cdot \Phi$ ), but also a part of the magnetic flux which embraces only part turns. That part of the magnetic flux (in the example shown in Figure 2) does not flow through the magnetic core and it represents a loss of magnetic energy for which it is called leakage flux (Fig. 3).

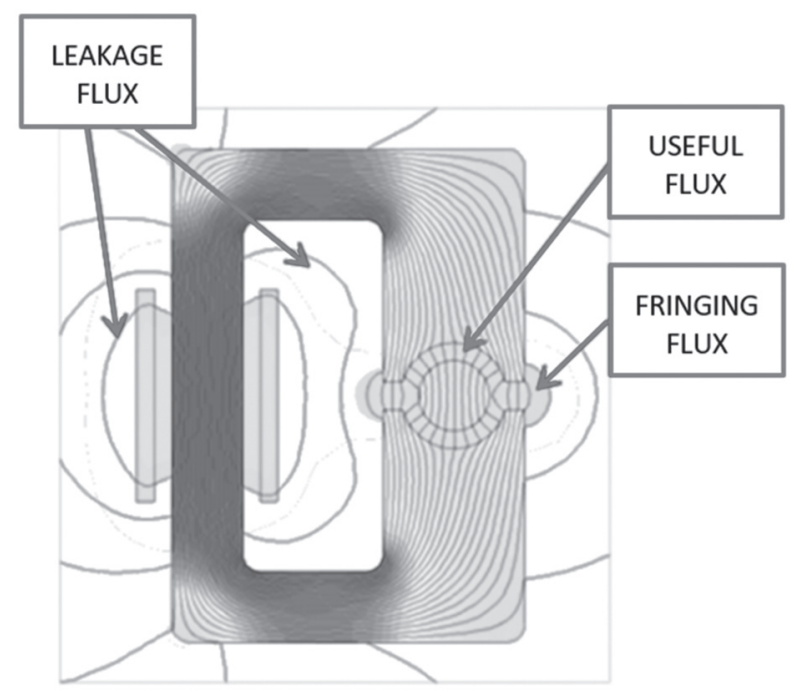

Fig. 3. A magnetic circuit with leakage flux and fringing flux that are not useful in energy conversion 
Another problem in the consideration of magnetic circuits are air gaps as interruptions of the looping magnetic core. In place of the air gap, the magnetic flux from the magnetic conductive area passes in the air which is not a good conductor of the magnetic flux. Also, the air cannot be shaped (as an iron core) so the magnetic flux through the air gap depends on many parameters but it primarily depends on the construction of the magnetic circuit.
Figure 3 shows the magnetic circuit designed to convert electrical energy from the coil into the magnetic energy in the rotor. The part of the magnetic flux which links all coil turns, passing through the core and through the air gap into the rotor and back, is called the useful magnetic flux.

\begin{tabular}{|c|c|c|c|c|c|c|}
\hline Flux density $|\mathrm{B}|$ & Air Coil & $L_{F_{e}} / L_{C_{u}}=1.0$ & $L_{F e} L_{C_{H}}=1.5$ & $L_{F_{e}} / L_{C_{\nu}}=2.0$ & $L_{F_{e}} / L_{C_{u}}=2.5$ & $L_{F_{e}} / L_{C_{M}}=3.0$ \\
\hline $0.8[T]$ & & & & & & \\
\hline $0.7[\mathrm{~T}]$ & & & & & & \\
\hline $0.6[\mathrm{~T}]$ & & & & & & \\
\hline $0.5[\mathrm{~T}]$ & & & & & & \\
\hline $0.4[\mathrm{~T}]$ & & & & & & \\
\hline $0.3[\mathrm{~T}]$ & & & & & & \\
\hline $0.2[\mathrm{~T}]$ & & & & & & \\
\hline $0.1[\mathrm{~T}]$ & & & & & & \\
\hline $0.0[\mathrm{~T}]$ & & & & & & \\
\hline $\begin{array}{l}\text { Coil Current } \\
\quad \mid[\mathrm{A}]\end{array}$ & 10.0 & 10.0 & 10.0 & 10.0 & 10.0 & 10.0 \\
\hline $\begin{array}{c}\text { Coil Voltage } \\
\text { U [V] }\end{array}$ & $1, .2$ & 1.82 & 1.82 & 1.82 & 1.82 & 1.82 \\
\hline $\begin{array}{l}\text { Flux Linkage } \\
\Psi \text { [mWb] }\end{array}$ & 0.212 & 1.00 & 1.28 & 1.43 & 1.52 & 1.58 \\
\hline $\begin{array}{c}\text { Inductance } \\
\mathrm{L}[\mu \mathrm{H}]\end{array}$ & 21.2 & 100 & 128 & 143 & 152 & 158 \\
\hline $\begin{array}{c}\text { Resistance } \\
\mathrm{R}[\Omega]\end{array}$ & 0.182 & 0.182 & 0.182 & 0.182 & 0.182 & 0.182 \\
\hline $\begin{array}{c}\text { Power Loss } \\
\text { P [W] }\end{array}$ & 18.2 & 18.2 & 18.2 & 18.2 & 18.2 & 18.2 \\
\hline
\end{tabular}

Fig. 4. Comparing magnetic fields of coils with the pole core of different length and tabular data of electrical coil data required to create a lumped model

Leakage flux shown in Figure 3 has several parts. It is shown that the magnetic flux embraces only coil turns. Also, there is a part of magnetic flux that passes through all coil turns, but because of core construction, it passes near the rotor and it is therefore not part of the useful flux (fringing flux).

For the purpose of understanding behavior of the magnetic field in the further analysis, a simple example of a coil and a pole core (Figure 4) will be considered. The influence of core length on the distribution of a magnetic field is shown by simultaneous display of the coil through which unchangeable current $I$ flows. The coil has $N$ turns and the length of the coil is designated as $L_{\mathrm{Cu}}$. The thickness of the coil and the magnetic core makes a negligible contribution in relation to the influ- ence of the length of the magnetic pole on the changes of the magnetic field.

The parameter ratio of core length compared to coil length increased from 1 to 3 , while the first image on the left in Figure 4 shows the distribution of the magnetic field of an air coil. With the same excitation (DC current), it can be seen in Table 1 that current parameters do not change.

However, increasing the length of the core is proportional to an increase in the value of magnetic flux density in the center of the coil. It could be concluded that reduced reluctance of the magnetic circuit and part of the magnetic flux which links the coil and the core becomes larger (an increase in a magnetic flux from the ends of the core). 
Flux density in the center of the coil in relation to core length

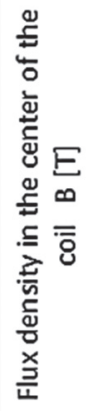

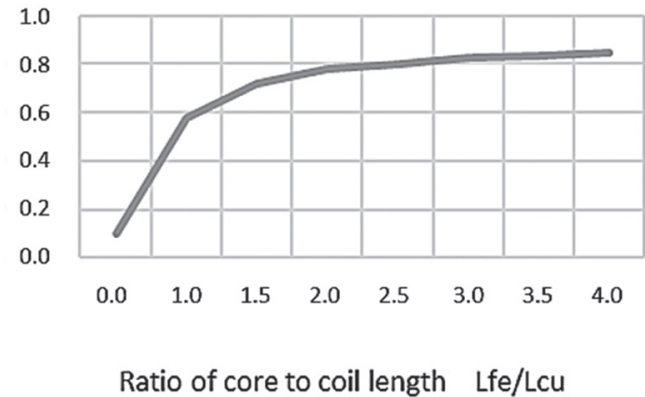

Fig. 5. Flux density in the center of the coil in relation to the ratio of core to coil length

The relations of these magnetic fluxes are inversely proportional in the case of parallel-connected reluctances. A further increase in core length by over 3 times the length of the coil does not significantly affect the increase in magnetic flux density, which is used as an indicator of the ratios between the amounts of reluctances.
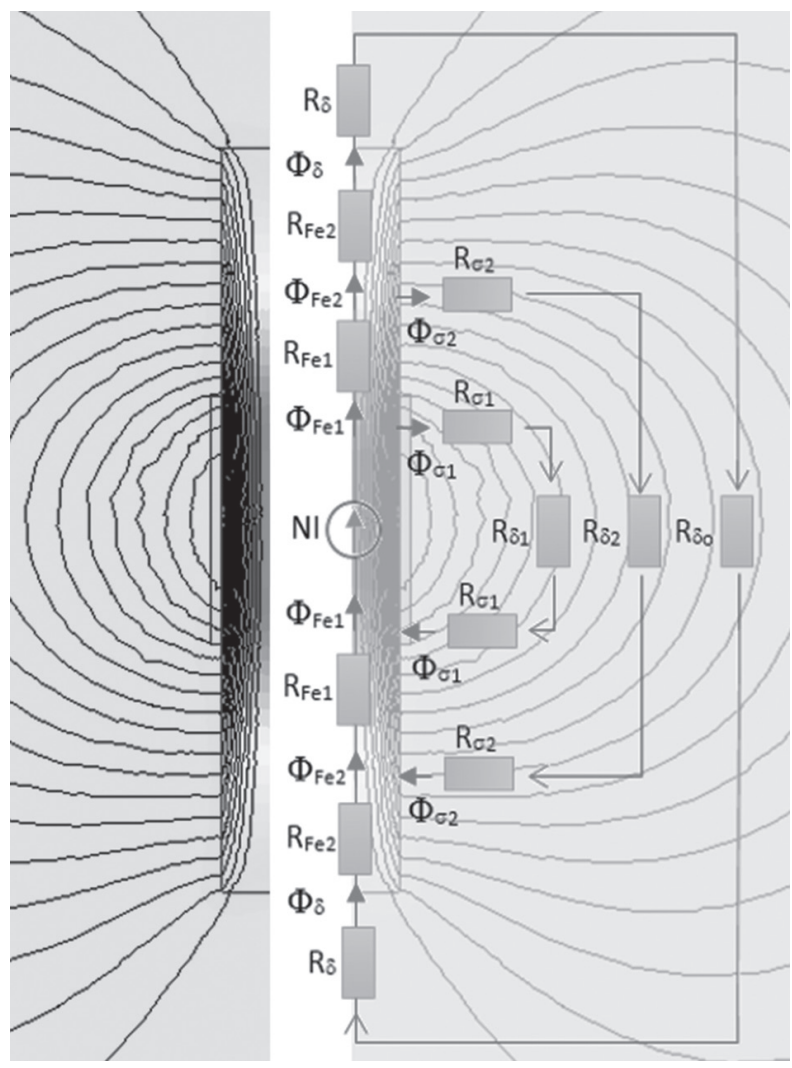

Fig. 6. Flux distribution in the example of $\mathrm{L}_{\text {core }}>\mathrm{L}_{\text {coil }}$ and the lumped model for concentrated parameters

In order to understand magnetic circuit modeling it is necessary to determine reluctance of certain parts of the magnetic circuit. The excitation $(N \cdot)$ in the center of the coil forces the magnetic flux $\left(\Phi \cdot R_{\mathrm{m}}=N \cdot\right)$, which is divided into a part that runs through the core and encompasses all turns of the coil $\Phi_{\mathrm{Fe} 1}$ (reluctance $R_{\mathrm{Fe})}$ ) and the part of the leakage magnetic flux $\Phi_{\sigma 1}$ (reluctance $R_{01}$ ) that closes over the air around the coil part (reluctance $R_{\delta 1}$ ). Part of the magnetic flux which has passed through the central part of the core is divided into a part that continues to pass through the core towards its end $\Phi_{\mathrm{Fe} 2}$ (reluctance $R_{\mathrm{Fe} 2}$ ) and a part of the magnetic flux emerging from the core $\Phi_{\mathrm{o} 2}$ (reluctance $R_{\mathrm{o} 2}$ ) and closes through the air to the other end of the core (reluctance $R_{\delta 0}$ ).

Finally, the remaining part of the magnetic flux that comes to the end of the core $\Phi_{\delta}$ (reluctance $R_{\delta}$ ) comes out and over the air it closes to the other end of the core (reluctance $R_{\delta 0}$ ).

It should be noted that no part of the above magnetic flux is to overlap, but they all match in parallel from the core to infinity.

Figure 7 shows a lumped model of the magnetic circuit consisting of the excitation branch and three parallel load branches. Although such complex magnetic circuit appears to be complicated for solving, it is possible by using a software program for numerical solution of electromagnetic fields (FEM - finite elements method), construction data, material properties and excitation, with the calculation yields of a magnetic flux of certain parts of the space, as well as magnetic voltage drops on certain parts of the way in which magnetic field lines are closed.

Following the analogous procedure, when the core and the coil are of equal length $\left(\mathrm{L}_{\text {core }}=\mathrm{L}_{\text {coil }}\right)$, magnetic field distribution has only two magnetic fluxes, and the equivalent model has only two parallel branches of the load. This means that the total reluctance of the magnetic circuit will be higher (the rule of a parallel resistor connection) so flux density in the center of the coil will be increasing for the same excitation ( $N \cdot I=$ const).
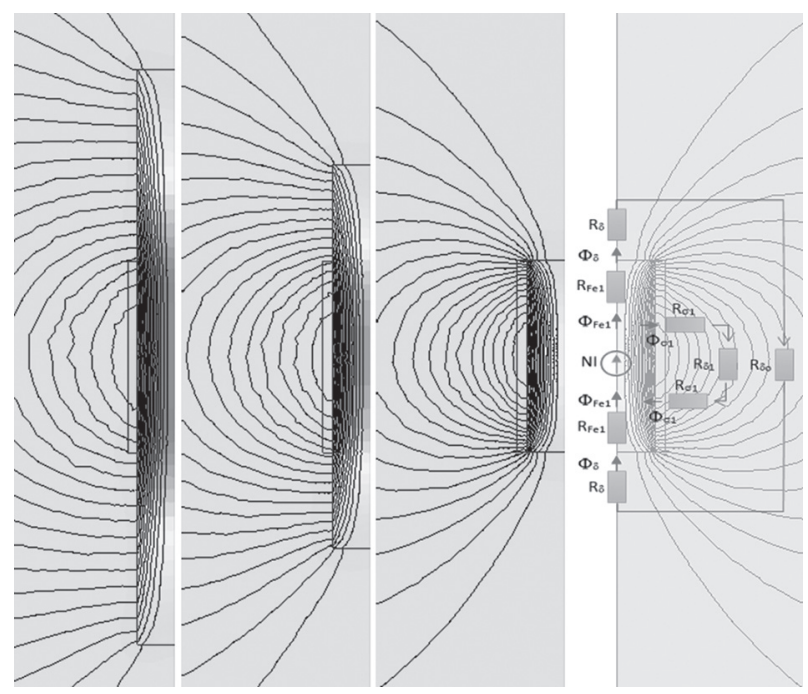

Fig. 7. Transient change of a magnetic field with respect to core length and reduction of the lumped model for the case $\left(\mathrm{L}_{\text {coil }}=\mathrm{L}_{\text {core }}\right)$.

In the case of an increased air gap between the coil and the core as the result, an additional part of leakage flux between coil turns and the core appears (Figure 8). Analogously to the previous consideration of the equiv- 
alent scheme, additional reluctance is introduced which eventually reduces flux density in the center of the core.
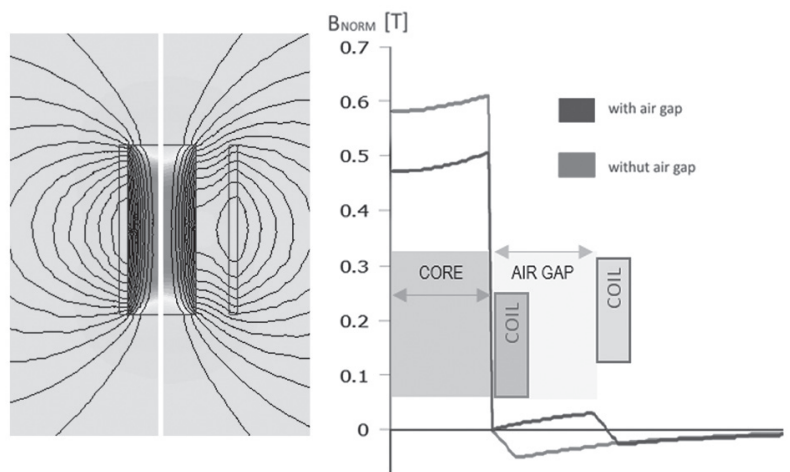

Fig. 8. Flux distribution in the example of increasing the distance between coils and the core

Analysis of changes in the distribution of the magnetic field in the case of an increased distance between the coil and the magnetic core for different core lengths indicates that previous considerations are applicable to this combination. Each of the previously mentioned phenomena have an impact on modifications to the equivalent lumped model, as shown in Figure 9.
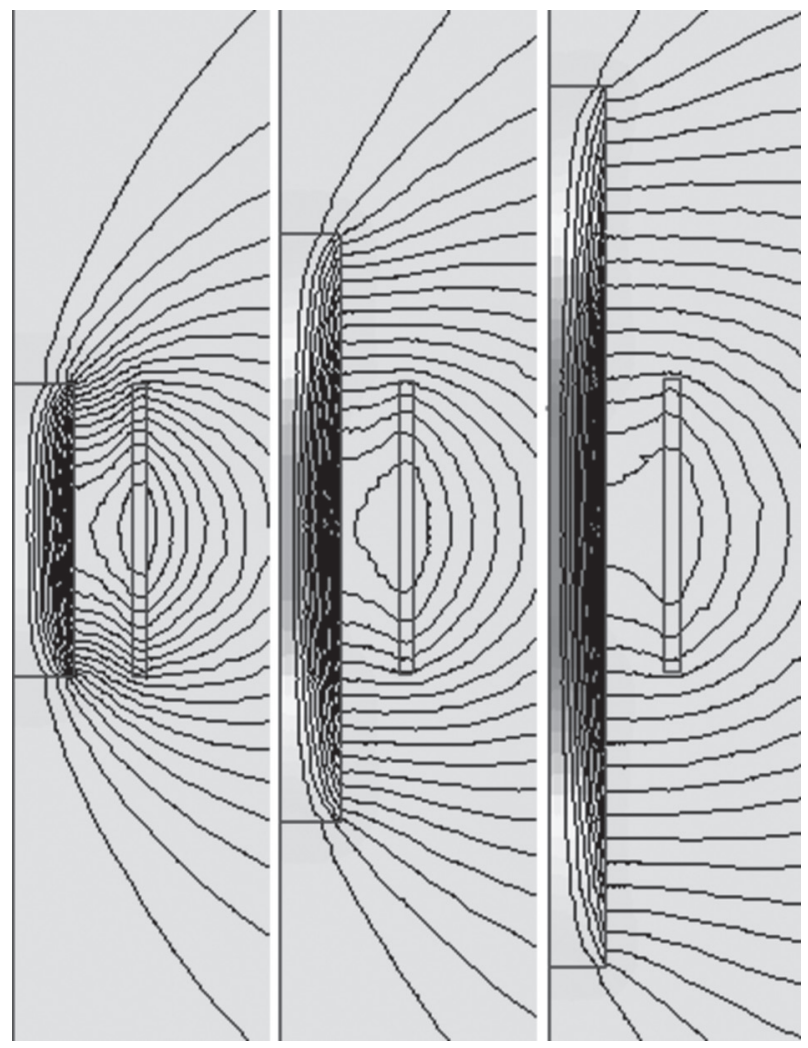

Fig. 9. Flux distribution in the example of the distance between coils and the core for different core lengths

\section{TRANSFORMER DESIGN WITH INCREASED LEAKAGE FLUX}

Previous considerations will be verified by constructing the educational transformer model with a modified coil in order to preserve space for measuring probes and to show the influence of an increasing leakage flux on transformer operation.

For this purpose, the core of the existing three-phase three-pillar transformers was used. New winding spools (3D printing shown in Figure 10) are made. In order to obtain parameters for the design of a newly expanded coil (Figure 11), first we tested the existing transformers for which $\mathrm{B}-\mathrm{H}$ magnetization characteristics of cores is recorded.

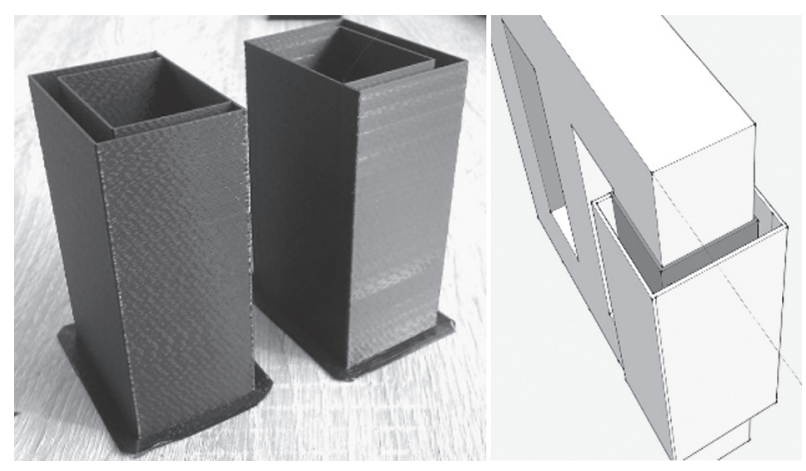

Fig. 10. New winding spools for increased leakage flux made by using a 3D printing technique

The existing models have been tested and calculated for the case of excitation with a time-invariant current. Further research will include numerical modeling and verification of models powered with alternating current, transient (FEM) analysis of the magnetic field and parameter calculation of the transformer lumped model.

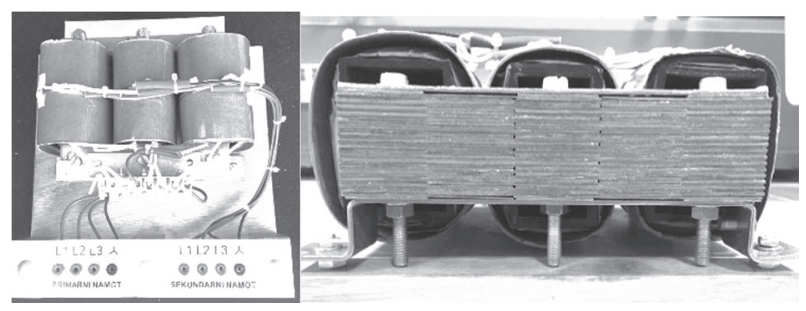

Fig. 11. Educational transformer unit designed to obtain the possibility of connecting the transformer in different ways (DY dyz) with space for measuring probes

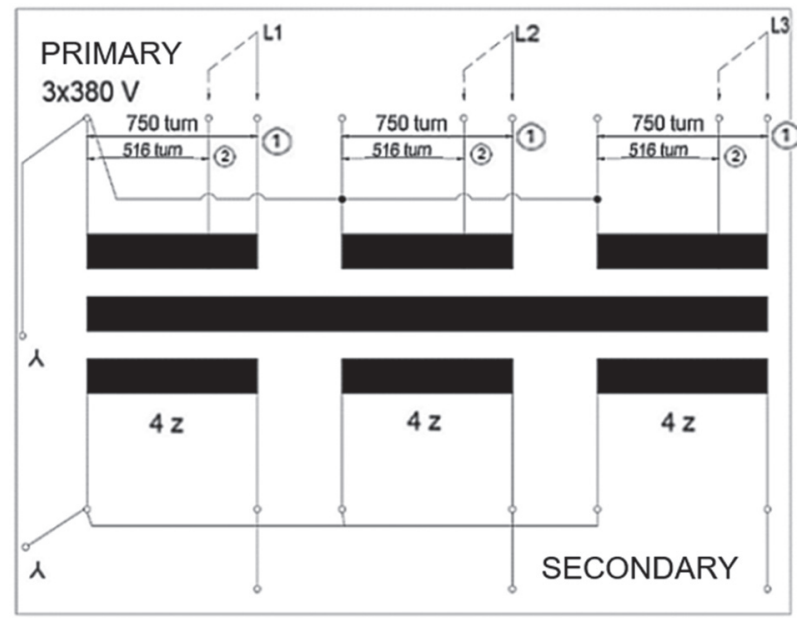

Fig. 12. A schematic wiring diagram of primary coils 


\section{CONCLUSION}

The study and research of magnetic fields in electromagnetic devices is a very complex problem. Usage of modern software tools for numerical calculation fields (FEM) considerably simplifies the calculation. Also, visualization of magnetic fields deepens the understanding of complex devices. Determination and influence of leakage fluxes are the major technical problems which often occur in practice. Getting students familiar with and educating them about the proposed models would provide a complete understanding of the theory of magnetic fields in devices.

\section{REFERENCES:}

[1] L. Rabins, "Transformer reactance calculations with digital", AIEE Transactions, Vol. 75, No. 1, 1956, pp. 261-267.

[2] P. I. Fergestad, T. Henriksen, "Inductances for the calculations in transformer", IEEE Transactions on Power Systems and Apparatus, 1974, pp. 510-517.

[3] D. J. Wilcox, W. G. Hurley, M. Conion, "Calculation of self and mutual impedances between sections of transformer windings", IEEE Proceedings C - Generation, Transmission and Distribution, Vol. 136, No. 5, 1989, pp. 308-314.

[4] F. De Leon, A. Semlyen, "Complete transformer model for electromagnetic transients", IEEE Transactions on Power Delivery, Vol. 9, No. 1, 1994, pp. 231-239.

[5] E. Rahimpour, J. Christian, K. Feser, H. Mohseni, "Modeling of a transformer winding for calculation of its transient function", Elektrie, Vol. 54, No. 1-2, 2000, pp. 18-30.

[6] D. Koprivanac, Ž. Hederić, "Calculation of singlephase transformer leakage reactance by finite elements method", Proceedings of the 18th International Conference "Electrical Engineering Symposium" Josip Lončar days, Šibenik, Croatia, 3-6 May 2009, pp. 158-163.

[7] S. Magdaleno-Adamei, J. C. Olivares-Galvan, E. Campero-Littlewood, R. Escarela-Perez, E. BlancoBrisseti, "Coil systems to generate uniform magnetic field volumes", Excerpt from the Proceedings of the COMSOL Conference 2010 Boston, USA.

[8] J. L. Kirschvink, "Uniform magnetic fields and double wrapped coil systems: Improved techniques for the design of bioelectromagnetic experiments", Bioelectromagnetics, Vol. 13, 1992, pp. 401-411.

[9] D. Cvetkovic, I. Cosic, "Modelling and design of extremely low frequency uniform magnetic field exposure apparatus for in vivo bioelectromagnetic studies", Proceedings of the 29th Annual International Conference of the IEEE Engineering in Medicine and Biology Society, Lyon, France, 22-26 August 2007, pp. 1675-1678.

[10] B. Shi, B. Farboud, R. Nuccitelli, R. Isseroff, "Powerline frequency electromagnetic fields do not induce changes in phosphorylation, localization, or expression of the 27-kilodalton heat shock protein in human keratinocytes", Environmental Health Perspectives, Vol. 111, No. 3, 2003, pp. 281-288.

[11] Ž. Hederić, M. Barukčić, N. Raičević, N. Cvetković, "Generating uniform magnetic field using Helmholtz coils system", Proceedings of Abstracts at the 31st Kando Conference 2015, Obudai University of Kando Kalman Electrical Engineering Faculty, Budapest, Hungary, 2015, Vol. 8, pp. 1-12.

[12] V. Reinbold, E. Vinot, L. Garbuio, L. Gerbaud, “Optimal sizing of an electrical machine using a magnetic circuit model: Application to a hybrid electrical vehicle", IET Electrical Systems in Transportation, Vol. 6, No. 1, 2016, pp. 27-33.

[13] D. K. Han, J. H. Chang, “Design of electromagnetic linear actuator using the equivalent magnetic circuit method", IEEE Transactions on Magnetics, 2016. (accepted for publication)

[14] M. Luo, D. Dujic, J. Allmeling, "Leakage flux modelling of multi-winding transformer using permeance magnetic circuit", Proceedings of the 2016 IEEE Applied Power Electronics Conference and Exposition, Long Beach, CA, USA 20-24 March 2016, pp. 1108-1114.

[15] A. M. Takbash, P. Pillay, "A modified analytical method for core losses calculation in magnetic laminations for a wide range of frequency and flux density", Proceedings of the 2015 IEEE International Electric Machines \& Drives Conference, Coeur d'Alene, ID, USA, 10-13 May 2015, pp. 11091114.

[16] Z. Haznadar, Ž. Štih, "Electromagnetism 1 and 2", Školska knjiga Zagreb, 1997. (in Croatian) 